\title{
THE SIMPLICITY OF CERTAIN NONASSOCIATIVE ALGEBRAS ${ }^{1}$
}

\section{RIMHAK REE}

Introduction. Let $V$ be an additively written elementary $p$-group of order $p^{n}$, where $n>1$, and let $\phi(u, v), \psi(u, v)$ be two alternate biadditive functions defined on $V$ and with values in a field $K$ of characteristic $p$. Let $k$ be a fixed nonzero element in $V$, and assume that the following two conditions are satisfied:

(0.1) If $\alpha, \beta$ are nonzero elements in $K$ then $\alpha \phi(u, v)+\beta \psi(u, v)=0$ for all $v$ implies $u=0$;

$(0.2) \phi(k, v)=0$ for all $v$.

If $\phi$ is identically zero, then the conditions (0.1)-(0.2) are equivalent to saying that $\psi$ is nondegenerate. Define an algebra $L$ over $K$ with basis

$$
\left\{e_{u} \mid u \in V, u \neq 0, u \neq k\right\}
$$

by the multiplication table:

$$
e_{u} e_{v}=\phi(u, v) e_{u+v}+\psi(u, v) e_{u+v+k},
$$

where we set $e_{0}=e_{k}=0$. The purpose of this note is to show that the algebra $L$ is simple if $p>2$.

An elementary computation shows that

$$
\begin{aligned}
\left(e_{u} e_{v}\right) e_{w} & +\left(e_{v} e_{w}\right) e_{u}+\left(e_{w} e_{u}\right) e_{v} \\
& =(\psi(u, v) \psi(k, w)+\psi(v, w) \psi(k, u)+\psi(w, u) \psi(k, v)) e_{u+v+w+2 k} .
\end{aligned}
$$

From this we may deduce easily that $L$ is a Lie algebra if and only if there exist two additive functions $f$ and $g$ on $V$ with values in $K$ such that

$$
\psi(u, v)=f(u) g(v)-f(v) g(u)
$$

for all $u, v$ in $V$. In the case where $\psi(u, v)$ is given as in (3), therefore, the algebra $L$ is a special case of the algebras considered by Richard Block [2] if the following conditions are satisfied: $V$ is the direct sum of subgroups $V_{1}$ and $V_{2}$ such that $k \in V_{2} ; \phi(u, v)=0$ for all $u \in V_{2}, v \in V ; \psi(u, v)=0$ for all $u \in V_{1}, v \in V$. If $\psi(u, v)$ cannot be expressed as in (3), then $L$ is of course not a Lie algebra. But it gives an interesting family of simple nonassociative algebras.

Received by the editors May 12, 1958.

1 This research was supported by the United States Air Force through the Air Research and Development Command under Contract No. AF 49(638)-152. 
1. The center of $L$. As a first step in proving the simplicity of $L$ we show that the center of $L$ is zero. Since $V$ is an elementary p-group, we may express $V$ as the direct sum of a subgroup $U$ and the subgroup generated by $k$. Then any element $x$ in $L$ can be written in the form

$$
x=\sum_{u \in U} \sum_{i=0}^{p-1} \alpha_{u i} e_{u+i k}
$$

with $\alpha_{u, i}$ in $K$, where $\alpha_{00}=\alpha_{01}=0$. For the element $x$ given by (1.1) define $x_{u}$ for each $u$ in $U$ by

$$
x_{u}=\sum_{i=0}^{p-1} \alpha_{u, i} e_{u+i k} .
$$

Then $x=\sum x_{u}$, and if $x$ is in the center of $L$ then for each $u$ in $U$ the element $x_{u}$ is also in the center of $L$. Therefore if the center of $L$ is not zero, then there must be in the center a nonzero element $x$ of the form

$$
x=\alpha_{0} e_{u}+\alpha_{1} e_{u+k}+\cdots+\alpha_{p-1} e_{u+(p+1) k},
$$

where $\alpha_{i} \in K$. For the element $x$ given by (1.2) define the length $\lambda(x)$ of $x$ to be the number of nonzero coefficients $\alpha_{i}$. Let the above $x$ be of minimal length among the nonzero central elements such that $x=x_{u}$ for some $u$. We consider the following two cases:

CASE I. $u=0$. Then $x$ is of the form

$$
x=\alpha_{2} e_{2 k}+\alpha_{3} e_{3 k}+\cdots+\alpha_{p-1} e_{(p-1) k} .
$$

Take an element $v \in U$ such that $\psi(k, v) \neq 0$. Then

$$
x e_{v}=\alpha_{2} \psi(2 k, v) e_{3 k+v}+\cdots+\alpha_{p-1} \psi((p-1) k, v) e_{v}=0 .
$$

Hence $\alpha_{2}=\cdots=\alpha_{p-1}=0 ; x=0$, a contradiction.

CASE II. $u \neq 0$. We have

$$
x e_{2 k}=\sum_{i=0}^{p-1} \alpha_{i} \psi(u+i k, 2 k) e_{u+(i+2) k}=0 .
$$

Since $u+(i+2) k \neq 0, k$ for all $i$, and since some $\alpha_{i} \neq 0$, we have $\psi(k, u)$ $=0$. Let $v \in V$ be such that $\psi(k, v) \neq 0$. Then for every $i, u+v+i k$ is neither zero nor $k$, since $\psi(k, u+v+i k) \neq 0$. Now we have

$$
x e_{v}=\sum_{i=0}^{p-1}\left(\alpha_{i} \phi(u, v)+\alpha_{i-1} \psi(u+(i-1) k, v)\right) e_{u+v+i k},
$$

where $\alpha_{-1}=\alpha_{p-1}$. Since $x e_{v}=0$, we have 


$$
\alpha_{i} \phi(u, v)+\alpha_{i-1} \psi(u+(i-1) k, v)=0
$$

for all $i$. If $w$ is an element such that $\psi(k, w)=0$ then $\psi(k, v+w) \neq 0$. Hence we may replace $v$ in (1.3) by $v+w$. Then it follows that (1.3) is true for all $v \in V$ and all $i$. Let $i=1$ in (1.3) and use (0.1). Then we have $\alpha_{0}=0$ or $\alpha_{1}=0$. Take an $i$ such that $\alpha_{i} \neq 0, \alpha_{i-1}=0$. Then from (1.3) it follows that

$$
\phi(u, v)=0
$$

for all $v$ in $V$. Let $j$ be such that $\alpha_{j}=0, \alpha_{j-1} \neq 0$. Then from (1.3) we have

$$
\psi(u+(j-1) k, v)=0
$$

for all $v$ in $V$. Also from (1.4) we have $\phi(u+(j-1) k, v)=0$ for all $v \in V$. Hence we have $u+(j-1) k=0$, a contradiction.

2. Minimal elements. For $x$ in $L$ denote by $\rho(x)$ the number of nonzero $x_{u}$, where $u \in U$. Let $M$ be a fixed nonzero ideal of $L$, and call a nonzero element $x$ in $M$ minimal if $\rho(x)$ is minimal. First we prove

Lemma 2.1. There exists a minimal element $x$ in $M$ for which $x_{0} \neq 0$.

Proof. We shall derive a contradiction by assuming that the lemma is not true. Let $x=\sum x_{u}$ be a minimal element. If there exists an element $u$ in $U$ such that $x_{u} \neq 0$ and $\psi(k, u) \neq 0$, then for any integer $j$ the element $u+j k$ is neither 0 nor $-k$. Hence we may consider the product $y=e_{-u-j k} x$. It is readily seen that $\psi(k, u) \neq 0$ implies that $y_{0} \neq 0$ for some $j$. Clearly $\rho(y) \leqq \rho(x)$ and $y \in M$. Hence it follows from our assumption that, for any minimal element $x, x_{u} \neq 0$ implies $\psi(k, u)=0$. Take an element $v$ in $V$ such that $\psi(k, v) \neq 0$. Then $x_{u} e_{v}=0$ for any minimal element $x$, because for $y=x e_{v}$ we have $\rho(y)$ $\leqq \rho(x)$ and if $y_{w} \neq 0$ then $w=u+v$, where $\psi(k, u)=0$, and hence $\psi(k, w) \neq 0$ which contradicts the conclusion obtained above. Now consider elements $v^{\prime}$ in $V$ such that $\psi\left(k, v^{\prime}\right)=0, v^{\prime} \neq 0, v^{\prime} \neq k$. Then $\psi\left(k, v+v^{\prime}\right) \neq 0$, and hence $x_{u} e_{v+v^{\prime}}=0$. Let

$$
x_{u}=\alpha_{0} e_{u}+\alpha_{1} e_{u+k}+\cdots+\alpha_{p-1} e_{u+(p-1) k} .
$$

Then $x_{u} e_{v}=0$ implies

$$
\alpha_{i} \phi(u, v)+\alpha_{i-1} \psi(u+(i-1) k, v)=0
$$

for all $i$, where we set $\alpha_{-1}=\alpha_{p-1}$, because $u+v+i k$ is neither zero nor $k$. Similarly we have

$$
\alpha_{i} \phi\left(u, v+v^{\prime}\right)+\alpha_{i-1} \psi\left(u+(i-1) k, v+v^{\prime}\right)=0
$$


and hence from (2.1) we have

$$
\alpha_{i} \phi\left(u, v^{\prime}\right)+\alpha_{i-1} \psi\left(u+(i-1) k, v^{\prime}\right)=0
$$

for all $i$, which shows that $x_{u} e_{v^{\prime}}=0$. Thus we have shown that $x$ belongs to the center of $L$. By the result in the first section, $x=0$. This is a contradiction. Thus Lemma 2.1 is proved.

Let $x$ be a minimal element in the ideal $M$ such that $x_{0} \neq 0$ and such that the number of nonzero terms in $x_{0}$ is as small as possible. Let

$$
x_{0}=\alpha_{2} e_{2 k}+\alpha_{3} e_{3 k}+\cdots+\alpha_{p-1} e_{(p-1) k} .
$$

Take $v \in V$ such that $\psi(k, v) \neq 0$, and consider $y=\left(x e_{v-k}\right) e_{-v-k}$. It is clear that either $y=0$ or $y$ is a minimal element in $M$, and that $y_{0}=\left(x_{0} e_{v-k}\right) e_{-v-k}$. We have

$$
y_{0}=-\psi(k, v)^{2} \sum_{i=2}^{p-1}(i-1) i \alpha_{i} e_{i k}
$$

Let $j, 2 \leqq j \leqq p-1$, be such that $\alpha_{2}=\cdots=\alpha_{j-1}=0, \alpha_{j} \neq 0$, and consider $z=-\psi(k, v)^{2}(j-1) j x-y$. It is clear that $\rho(z) \leqq \rho(x)$, and that the number of nonzero terms in $z_{0}$ is smaller than that of $x_{0}$. Hence by the assumption on $x$ we have $z=0$. Then for any $t$ such that $\alpha_{t} \neq 0$, we have $j(j-1) \equiv t(t-1)(\bmod p)$. Hence $t=j$ or $t=p-j+1$. Therefore $x_{0}$ consists of only one nonzero term or is of the form

$$
x_{0}=\alpha e_{j k}+\beta e_{(p-j+1) k},
$$

where $\alpha, \beta$ are nonzero elements in $K$. We rule out the second possibility as follows: We may assume without loss of generality that $2 \leqq j<(p+1) / 2$. Let again $v$ be such that $\psi(k, v) \neq 0$, and consider $y^{\prime}=\left(x e_{v}\right) e_{-v+(j-2) k}$. Clearly $\rho\left(y^{\prime}\right) \leqq \rho(x)$ and $y_{0}^{\prime}=\left(x_{0} e_{v}\right) e_{-v+(j-2) k}$. We have

$$
x_{0} e_{v}=\psi(k, v)\left(j \alpha e_{v+(j+1) k}+(1-j) \beta e_{v+(2-j) k}\right) .
$$

Since $e_{w} e_{-w}=0$ for any $w$, we have from the above

$$
y_{0}^{\prime}=-\psi(k, v)^{2} j(2 j-1) \alpha e_{2 j k} \neq 0 .
$$

Thus we have proved that $x_{0}$ consists of a single nonzero term. Let $x=e_{j k}+\sum_{u \neq 0} x_{u}$, where $2 \leqq j<p-1$. Take $v \in V$ such that $\psi(k, v) \neq 0$, and consider $x^{\prime}=\left(x e_{v}\right) e_{-v-k}$. We have

$$
x^{\prime}=-j^{2} \psi(k, v)^{2} e_{(j-1) k}+\sum\left(x_{u} e_{v}\right) e_{-v-k} .
$$

Hence we may assume that $j=p-1 ; x=e_{-k}+\sum_{u \neq 0} x_{u}$. Then from the above we have $x_{0}^{\prime}=0$. Hence $\rho\left(x^{\prime}\right)<\rho(x) ; x^{\prime}=0 ;\left(x_{u} e_{v}\right) e_{-v-k}=0$ for all nonzero $u$ in $U$. Similarly $\left(x_{u} e_{v}\right) e_{-v}=0$ for all $u \in U$. Let 


$$
x_{u} e_{v}=\sum_{i=0}^{p-1} \beta_{i} e_{u+v+i k} .
$$

Then $\left(x_{u} e_{v}\right) e_{-v}=0$ implies

$$
\beta_{i} \phi(u,-v)+\beta_{i-1} \psi(u+(i-1) k,-v)=0
$$

for all $i ;\left(x_{u} e_{v}\right) e_{-v-k}=0$ implies

$$
\beta_{i} \phi(u,-v)+\beta_{i-1} \psi(u+(i-2) k,-v-k)=0
$$

for all $i$. Hence it follows that $\beta_{i-1} \psi(u+v, k)=0$ for all $i$ whenever $\psi(v, k) \neq 0$. Using $2 v$ instead of $v$ we have $\beta_{i-1} \psi(u+2 v, k)=0$. Therefore $\beta_{i}=0$ for all $i$ (note that $\beta_{-1}=\beta_{p-1}$ ). Thus we have shown that $x_{u} e_{v}=0$ whenever $\psi(k, v) \neq 0$. Then by proceeding as in the proof of Lemma 2.1 we may conclude that $x_{u}=0$ for all nonzero $u$ in $U$. Thus it is shown that the ideal $M$ contains $e_{-k}$.

3. The simplicity of $L$. Let $M$ be an arbitrary nonzero ideal of $L$. By the preceding section $M$ contains $e_{-k}$, and since $e_{-k} e_{v}=-\psi(k, v) e_{v}$, $M$ also contains $e_{v}$ for every $v$ such that $\psi(k, v) \neq 0$. Consider now an element $u$ in $V$ such that $\psi(k, u)=0, u \neq 0, u \neq k, u \neq-k$.

CASE I. $\psi(u, w)=0$ for all $w$ in $V$. In this case take an element $v$ in $V$ such that $\psi(k, v) \neq 0$. Since $e_{-v}$ is in $M, e_{u+v} e_{-v}=\phi(u,-v) e_{u}$ is in $M$. Hence if $e_{u}$ is not in $M$, then $\phi(u, v)=0$ for all $v$ such that $\psi(k, v)$ $\neq 0$. Let $\psi\left(k, v^{\prime}\right)=0$. Then $\psi\left(k, v+v^{\prime}\right) \neq 0 ; \phi\left(u, v+v^{\prime}\right)=0 ; \phi\left(u, v^{\prime}\right)=0$. Thus $\psi(u, w)=\phi(u, w)=0$ for all $w$ in $V$. Then by $(0.1)$ we have $u=0$, a contradiction. In this case, therefore, $e_{u}$ is contained in $M$.

CASE II. $\psi(u, w) \neq 0$ for some $w$ in $V$. In this case there exists $v \in V$ such that $\psi(u, v) \neq 0, \psi(k, v) \neq 0$, for if $\psi(u, v)=0$ for all $v$ such that $\psi(k, v) \neq 0$, then it may be proved as in Case I that $\psi(u, w)=0$ for all $w$ in $V$. Take an arbitrary $v$ such that $\psi(k, v) \neq 0$. Then, since $e_{-v-k}$ is in $M, e_{u+v} e_{-v-k}$ is also in $M$. We have

$$
e_{u+v} e_{-v-k}=\phi(u,-v) e_{u-k}+\psi(u,-v) e_{u} \in M .
$$

If $u=2 k$ then from (3.1) it follows that $e_{u}$ is in $M$, since there exists $v$ such that $\psi(u, v) \neq 0, \psi(k, v) \neq 0$. Therefore we may assume $u \neq 2 k$. If $e_{u}$ is not in $M$ then from (3.1) it follows that there exist $\alpha \neq 0$ and $\beta$ in $K$ such that

$$
\alpha \phi(u, v)+\beta \psi(u, v)=0
$$

for all $v$ for which $\psi(k, v) \neq 0$. If $\psi\left(k, v^{\prime}\right)=0$ then $\psi\left(k, v+v^{\prime}\right) \neq 0$, and hence we may replace $v$ in (3.2) by $v+v^{\prime}$. From this we may infer that (3.2) is true for all $v \in V$. Since $u \neq 0,(0.1)$ now implies that $\beta=0$. Then from (3.2) it follows that $e_{u} \in M$, a contradiction. Thus 
we have shown that every basis element is contained in $M ; M=L$. Thus the simplicity of $L$ is proved.

4. Remarks. The algebra $L$ considered above may be generalized in the obvious way if we use $m+1$ biadditive functions $\phi_{0}, \phi_{1}, \cdots, \phi_{m}$ instead of $\phi$ and $\psi$, and $m$ constant elements $k_{1}, \cdots, k_{m}$ instead of $k$ (see [2] for instance). The author, however, has been unable to obtain any result in this direction. Finally we remark that the Lie algebra $L$ and its generalizations mentioned above are all obtained as special cases of Lie algebras constructed as follows: Let $\mathfrak{A}$ be the group algebra over $K$ of an elementary $p$-group, and let $\left(D_{1}, \cdots, D_{r}\right)$ be an orthogonal system (see [3] for definition) of derivations of $\mathfrak{A}$. Let $a_{1}, \cdots, a_{r}$ in $\mathfrak{A}$ be such that $D_{i} a_{j}=D_{j} a_{i}$ for all $i, j$. Suppose that $r^{2}$ elements $a_{i j},(i, j=1, \cdots, r)$, in $\mathfrak{A}$ satisfy the following conditions:

$$
a_{i j}+a_{j i}=0 ; \quad a_{i i}=0
$$

for all $i$ and $j$;

$$
\sum_{s=1}^{r}\left(a_{i s} D_{s} a_{j k}+a_{j s} D_{s} a_{k i}+a_{k s} D_{s} a_{i j}\right.
$$

$$
\left.+a_{i s} a_{s} a_{j k}+a_{j s} a_{s} a_{k i}+a_{k s} a_{s} a_{i j}\right)=0,
$$

for all $i, j$, and $k$.

Then an elementary computation shows that the set $L$ of derivations of $\mathfrak{A}$ of the form

$$
E_{f}=\sum_{s, t} a_{s t}\left(D_{t} f-a_{t} f\right) D_{s}
$$

where $f$ runs over $\mathfrak{A}$, forms a subalgebra of the derivation algebra of I. We have $\left[E_{f}, E_{\imath}\right]=E_{h}$, where

$$
h=\sum_{s, t} a_{s t}\left(D_{t} f-a_{t} f\right)\left(D_{s} g-a_{s} g\right) .
$$

The Lie algebras considered in this note and those in [2] can be obtained by taking some special values of $a_{i}, a_{i j}$. In case $\operatorname{det}\left(a_{i j}\right)$ is a unit in $\mathfrak{A}$, we may simplify the conditions $(4.1)-(4.2)$ by using $\left(b_{i j}\right)$ $=\left(a_{i j}\right)^{-1}$. It may be seen easily that (4.1)-(4.2) are equivalent to the following:

$$
\begin{gathered}
b_{i j}+b_{j i}=0 ; \quad b_{i i}=0 \\
\left(D_{i} b_{j k}-a_{i} b_{j k}\right)+\left(D_{j} b_{k i}-a_{j} b_{k i}\right)+\left(D_{k} b_{i j}-a_{k} b_{i j}\right)=0
\end{gathered}
$$

for all $i, j$, and $k$. 


\section{REFERENCES}

1. A. A. Albert and M. S. Frank, Simple Lie algebras of characteristic p, Rend. Sem. Mat. Univ. e Politech. di Torino vol. 14 (1955) pp. 117-139.

2. Richard Block, New simple Lie algebras of prime characteristic, Trans. Amer. Math. Soc. vol. 89 (1958) pp. 421-449.

3. S. A. Jennings and Rimhak Ree, On a family of Lie algebras of characteristic $p$, Trans. Amer. Math. Soc. vol. 84 (1957) pp. 192-207.

The University of British Columbia, Vancouver, B. C.

\section{A NOTE ON COMPLETE BOOLEAN ALGEBRAS}

\section{R. S. PIERCE}

1. Introduction. Among commutative rings, Boolean algebras stand just below fields in simplicity of structure. In contrast, little is known concerning their classification. The purpose of this paper is to present a decomposition theorem for complete Boolean algebras, which, in a small way, simplifies the classification problem. As an illustration of how this decomposition theorem can be used, it is shown that an infinite cardinal number $\boldsymbol{N}$ can be the power of a complete Boolean algebra if and only if $\boldsymbol{\aleph}^{\boldsymbol{N}_{0}}=\boldsymbol{N}$.

The words "complete Boolean algebra" will henceforth often be abbreviated C.B.A. Similarly, B.A. stands for Boolean algebra. The join, meet and complement operations in a B.A. are denoted $V$, $\wedge$ and $\left({ }^{\prime}\right)$. Inclusion is indicated by $\leqq$. Also 0 and $u$ respectively stand for the zero and unit elements of a B.A. The least upper bound of a subset $A$ of a C.B.A. is designated l.u.b. $A$.

The relation of isomorphism between Boolean algebras is denoted $\cong$. If $B$ is a B.A. and $a \in B$, then $B_{a}$ will stand for the set $\{b \in B \mid b \leqq a\}$. If $a \neq 0$, then $B_{a}$ is a B.A. (which is complete if $B$ is complete) with the join, meet and zero inherited from $B$ and with complementation relative to $a$. That is, the complement of $b \leqq a$ in $B_{a}$ is $b^{\prime} \wedge a$. A Boolean algebra $B$ is called homogeneous if $B_{a} \cong B$ for all $a \neq 0$ in $B$. A useful property of C.B.A.'s is they satisfy the Schroeder-Bernstein theorem: if $B \cong \bar{B}_{a}$ and $\bar{B} \cong B_{a}(a \in B, \bar{a} \in \bar{B})$, then $B \cong \bar{B}$. This fact is proved in various places, perhaps the most accessible of which is [5, Theorem 1.31].

The direct union of a set $\left\{B_{\sigma} \mid \sigma \in S\right\}$ of B.A.'s is defined in the usual way (see $[1, \mathrm{p}$. viii $]$ ). This direct union will be denoted $\sum_{\sigma \in S} B_{\sigma}$. There is a useful internal characterization of direct unions of C.B.A.'s.

Received by the editors May 2, 1958. 